\title{
NEON NIST data science evaluation challenge: methods and results of team FEM
}

\author{
Michele Dalponte ${ }^{\text {Corresp., }}{ }^{1}$, Lorenzo Frizzera ${ }^{1}{ }^{,}$Damiano Gianelle ${ }^{1}$ \\ ${ }^{1}$ Dept. of Sustainable Agro-Ecosystems and Bioresources, Research and Innovation Centre, Fondazione Edmund Mach, San Michele all'Adige, Trento, Italia \\ Corresponding Author: Michele Dalponte \\ Email address: michele.dalponte@fmach.it
}

An international data science challenge, called NEON NIST data science evaluation, was set up in autumn 2017 with the goal to improve the use of remote sensing data in ecological applications. The competition was divided into three tasks: 1) segmentation of tree crowns; 2) data alignment; and 3) tree species classification. In this paper the methods and results of team FEM in the NEON NIST data science evaluation challenge are presented. The individual tree crown (ITC) segmentation (Task 1 of the challenge) was done using a region growing method applied to a near-infrared band of the hyperspectral images. The optimization of the parameters of the segmentation algorithm was done in a supervised way on the basis of the Jaccard score using the training set provided by the organizers. The alignment (Task 2 ) between the segmented ITCs and the ground measured trees was done using an Euclidean distance among the position, the height, and the crown radius of the ITCs and the ground trees. The classification (Task 3 ) was performed using a Support Vector Machine classifier applied to a selection of the hyperspectral bands. The selection of the bands was done using a Sequential Forward Floating Selection method and the Jeffries Matusita distance. The results in the three tasks were very promising: team FEM ranked first in Task 1 and 2, and second in Task 3. The segmentation results showed that the proposed approach segmented both small and large crowns. The alignment was correctly done for all the test samples. The classification results were good, even if the accuracy was biased towards the most represented species. 


\section{NEON NIST data science evaluation challenge: methods and}

\section{2 results of team FEM}

3 Michele Dalponte, Lorenzo Frizzera, and Damiano Gianelle

4 Dept. of Sustainable Agro-ecosystems and Bioresources, Research and Innovation Centre,

5 Fondazione E. Mach, Via E. Mach 1, 38010 San Michele all'Adige (TN), Italy.

6

7 Corresponding Author:

8 Michele Dalponte

9 Via E. Mach 1, 38010 San Michele all'Adige (TN), Italy

10 Email address: michele.dalponte@fmach.it

11

12 
13 Abstract

14 An international data science challenge, called NEON NIST data science evaluation, was set up 15 in autumn 2017 with the goal to improve the use of remote sensing data in ecological applications. The competition was divided into three tasks: 1) segmentation of tree crowns; 2) data alignment; and 3) tree species classification. In this paper the methods and results of team FEM in the NEON NIST data science evaluation challenge are presented. The individual tree crown (ITC) segmentation (Task 1 of the challenge) was done using a region growing method applied to a near-infrared band of the hyperspectral images. The optimization of the parameters of the segmentation algorithm was done in a supervised way on the basis of the Jaccard score using the training set provided by the organizers. The alignment (Task 2) between the segmented ITCs and the ground measured trees was done using an Euclidean distance among the position, the height, and the crown radius of the ITCs and the ground trees. The classification (Task 3) was performed using a Support Vector Machine classifier applied to a selection of the hyperspectral bands. The selection of the bands was done using a Sequential Forward Floating Selection method and the Jeffries Matusita distance. The results in the three tasks were very promising: team FEM ranked first in Task 1 and 2, and second in Task 3. The segmentation results showed that the proposed approach segmented both small and large crowns. The alignment was correctly done for all the test samples. The classification results were good, even if the accuracy was biased towards the most represented species.

\section{Introduction}

The NEON NIST data science evaluation challenge (Marconi et al., 2018) was an international competition with the goal to challenge international scientists on three tasks that are 
central in converting remote sensing images into vegetation diversity and structure information traditionally collected by ecologists: 1) individual tree crown (ITC) segmentation, for identifying the location and size of individual trees; 2) alignment to match ground truth data on trees with remote sensing; and 3) species classification to identify trees to species.

There is a large amount of literature about crown segmentation (e.g. Popescu, Wynne \& Nelson, 2003; Lee \& Lucas, 2007; Ene, Næsset \& Gobakken, 2012; Hung, Bryson \& Sukkarieh, 2012; Ferraz et al., 2012; Duncanson et al., 2015), and there have been many studies comparing segmentation methods on different data types (Ke \& Quackenbush, 2011; Vauhkonen et al., 2012; Eysn et al., 2015; Dalponte et al., 2015b). Many papers focus on light detection and ranging (LiDAR) data as these remote sensing data are very common in the forestry and ecology domains. Some studies exist on methods for crown segmentation of camera images, while fewer studies exist on segmentation of hyperspectral data (Dalponte et al., 2014).

The alignment to match ground truth data of trees with remote sensing was never explored in specific papers and usually only briefly mentioned on papers devoted to crown segmentation. This fact makes alignment very subjective because different approaches are used in every crown segmentation paper, and the alignment is adapted to the data used in the specific work.

Tree species classification with remote sensing data is a widely covered topic by the scientific literature (Fassnacht et al., 2016). The first studies on this topic were focusing on large categories of species as they were done using satellite multispectral data, but since the 2000s with the availability of airborne hyperspectral data many studies focused on the separation of tree species (Dalponte, Bruzzone \& Gianelle, 2012; Dalponte et al., 2013; Budei et al., 2017). Indeed, hyperspectral data due to their dense sampling of the spectral signatures can separate many 
58

59

60

61

62

63

64

65

66

67

68

69

70

71

72

73

74

75

76

77

different species with high level of accuracy. Moreover, the advances in the remote sensing community on development of hyperspectral image classifiers, and on band selection and reduction have significantly improved the possibility to detect tree species.

The objective of this paper is to present the methods and results of team FEM in the NEON NIST data science evaluation challenge. The FEM team belongs to the Forest Ecology and Biogeochemical cycles unit of the Research and Innovation Centre of the Edmund Mach Foundation in Italy. The research activities of the Forest ecology and Biogeochemical Cycles unit are focused on the interactions between the vegetation canopy and the atmosphere's chemicalphysical layer in addition to the soil structure and functionality. In particular, energy and matter (carbon, water, nitrogen) fluxes between the atmosphere and the biosphere are analysed and models simulating vegetation systems and turbulent and radiative transfer are used. These data are up-scaled at a regional level to obtain a carbon balance integrating ground and remote sensing data bases. The remote sensing team of the unit is specialized in LIDAR and hyperspectral image processing both from airborne and satellite sensors, on the forest domain.

\section{Materials}

For a detailed description of the data used we recall to (Marconi et al., 2018). The data from NEON included the following data products: 1) Woody plant vegetation structure (NEON.DP1.10098); 2) Spectrometer orthorectified surface directional reflectance - flightline (NEON.DP1.30008); 3) Ecosystem structure (NEON.DP3.30015); and High-resolution orthorectified camera imagery (NEON.DP1.30010). 
79

80

81

82

83

\subsection{Task 1: segmentation}

The ITCs segmentation was performed on the hyperspectral data using the algorithm presented in (Dalponte et al., 2015b). In greater detail the steps of the segmentation method were:

1. the hyperspectral band closest to $810 \mathrm{~nm}$ was selected for the segmentation;

2. the normalized difference vegetation index (NDVI) was computed for each pixel, and all the pixels in the band selected at step 1 having NDVI below 0.6 were masked;

3. seeds points $S=\left\{s_{1}, \ldots, s_{N}\right\}$ was defined using a moving window. An image pixel $H(x, y)$ was a seed point if:

$$
H(x, y) \in S \text { if } H(x, y)=\max (\text { moving window })
$$

4. initial regions were defined starting from the seed points. A label map $L$ was defined:

$$
\left\{\begin{array}{l}
L_{i, j}=k \text { if } H(i, j) \in S \\
L_{i, j}=0 \text { if } H(i, j) \notin S
\end{array}\right.
$$

5. starting from $L$, regions grew according to the following procedure:

a. a label map point $L_{i, j} \neq 0$ was considered and its neighbor pixels $(N P)$ in the image were taken:

$$
N P=\{H(i, j-1) ; \mathrm{H}(i-1, j) ; \mathrm{H}(i, j+1) ; \mathrm{H}(i+1, j)\}
$$

b. a neighbor pixel $N P\left(i^{\prime}, j^{\prime}\right)$ was added to the region $n$ if:

$$
N P\left(i^{\prime}, j^{\prime}\right) \in\left\{\begin{array}{l}
\operatorname{dist}\left(N P\left(i^{\prime}, j^{\prime}\right), s_{n}\right)<\text { DistMax } \\
N P\left(i^{\prime}, j^{\prime}\right)>\left(s_{n} * \text { PercThresh }\right) \\
L_{i, j^{\prime}} \neq 0
\end{array}\right.
$$

where PercThresh $\in(0 ; 1)$, and DistMax $>0$; 
94

95

c. this procedure was iterated over all pixels that have $L_{i, j} \neq 0$, and was repeated until no pixels were added to any region;

6. from each region in $L$ the central coordinates of each pixel were extracted, and a $2 \mathrm{D}$ convex hull was applied to these points;

7. the resulting polygons were the final ITCs.

The raster image used in this paper was the hyperspectral band at $810 \mathrm{~nm}$, already used in previous studies for this purpose (Clark, Roberts \& Clark, 2005; Dalponte et al., 2014). The parameters of the segmentation (i.e. the size of the moving window, PercThresh, and DistMax) were optimized in a supervised way using a training set made available by the organizers of the challenge: the set of parameters that provided the highest Jaccard score (Real \& Vargas, 1996) on the training set was chosen. The parameters used for the delineation on the test set were: a moving window a size of $3 \times 3$ pixels, a PercThresh of 0.4 , and a DistMax of 4 . The implementation used is the one in itcIMG of the itcSegment $\mathrm{R}$ package (Dalponte, 2016).

\subsection{Task 2: alignment}

The alignment between ground measured trees and the delineated ITCs was done using a four step procedure: 1) prediction of missing ground measured crown radius; 2) prediction of missing ITC heights; 3) linking ITCs and ground measured trees using an Euclidean distance based on X and $\mathrm{Y}$ coordinates, and height and crown radius; and 4) visual inspection of the results.

The crown radius of ground measured trees, for which this attribute was not measured on the ground, was predicted using a relationship linking the field measured crown radius $\left(R_{\text {FIELD }}\right)$ with the tree height $\left(H_{F I E L D}\right)$ and the stem diameter $\left(D_{F I E L D}\right)$ : 


$$
R_{\text {FIELD }}=a \times\left(H_{\text {FIELD }} \times D_{\text {FIELD }}\right)^{b}
$$

115 Eqn. 5 was fitted using the function $n l s$ of the package stats of the R software (R Development

116 Core Team, 2008).

117 The height of the ITCs, for which this attribute was missing, was predicted using a

118 relationship linking the ITCs height $\left(H_{\text {ITC }}\right)$ and the ITCs crown radius $\left(R_{I T C}\right)$ :

$$
H_{I T C}=a \times R_{I T C}^{b}
$$

119 Eqn. 6 was fitted using the function $n l s$ of the package stats of the R software (R Development 120 Core Team, 2008).

Each ITC was linked to the closest ground measured tree according to the Euclidean 122 distance between their position and their attributes (height, and crown radius):

$$
D=\sqrt{\left(X_{I T C}-X_{F I E L D}\right)^{2}+\left(Y_{I T C}-Y_{F I E L D}\right)^{2}}+\sqrt{\left(H_{I T C}-H_{F I E L D}\right)^{2}+\left(R_{I T C}-R_{F I E L D}\right)^{2}}
$$
After the linking, a visual inspection of the results on a GIS software was done and some trees were manually realigned.

\section{Task 3: classification}

126 The classification of the tree species was done with a four step procedure: 1) data normalization;

127 2) feature selection; 3) classification; and 4) aggregation.

Data normalization was done to ensure that the pixel values were uniformly distributed across all the crowns. Each pixel value was divided by the sum of the values of that pixel in all

130 the bands (Yu et al., 1999). In this way, we reduced the difference in radiance due to the fact that 131 the samples are distributed on multiple images. 
The feature selection is necessary in order to select only the bands that are useful to 133 separate the analysed species. A feature selection method is made up of a searching strategy and 134 a separability criterion. In this study, the search strategy we used was the Sequential Forward 135 Floating Selection (SFFS) (Pudil, Novovičová \& Kittler, 1994), and the separability criterion

136

137 138 139

was the Jeffries Matusita distance (Bruzzone, Roli \& Serpico, 1995). These methods were used successfully in many previous studies (Dalponte, Bruzzone \& Gianelle, 2008, 2012, Dalponte et al., 2009, 2013, 2014). The feature selection was applied on the training data, and we used the function varSelSFFS in the R package varSel.

The classification was performed using a Support Vector Machine (SVM) classifier, having as input the features selected at step 2 and the value of the CHM corresponding to each ITC. We used the SVM implemented in the R package kernlab.

The predicted species labels of each pixel were aggregated at crown level with a majority rule.

\section{Results}

\subsection{Task 1: segmentation}

The Jaccard score for the delineated ITCs over all the plots was 0.3402. The overall confusion matrix $(\mathrm{OCM})$ is showed in Table 1. To analyze the performance over each plot, the confusion matrix for each plot was visualized as a bar chart (see Figure 1). The Jaccard score by crown area is shown in Figure 2. Variability in the crown size did not change the Jaccard score, showing that the method used is behaving in the same way for all crown sizes. The top- 6 best and worst delineations of the system are shown in Figure 3 and 4, respectively. 


\subsection{Task 2: alignment}

154

All the test ITCs were aligned with the respective ground measured tree.

155

156

157

158

159

160

161

162

163

164

165

166

167

168

169

170

171

172

173

174

\subsection{Task 3: classification}

In Table 2 a summary of the overall performances is provided. Performance metrics at the class level are also shown: accuracy and specificity (Figure 5), F1 score (Figure 6), precision (Figure 7), and recall (Figure 8). From the overall performances, it is clear that the classification method used was effective, as all the performance metrics are quite good. Looking at the results per class (Table 3) it can be seen that for some classes the performance metrics are really low (e.g. ACRU), while others are really good (e.g. PIPA).

\section{Discussion}

Team FEM ranked first for Task 1. As explained in the methods, we chose to segment a hyperspectral band instead of the LiDAR point cloud. This choice was motivated by the fact that looking at the training ITCs provided by the organizers, the hyperspectral data seemed more suitable for this task. The comparison of results across teams showed that the FEM approach outperforms the other approaches in the delineation of the small trees, while it was less efficient for the large trees. This is due to the fact that we decided to use a small moving window ( $3 \times 3)$. The use of a variable size moving window, like the one that is implemented for LiDAR data in the itcSegment library and used in (Dalponte et al., 2018), would have probably improved the final results. The segmentation method used was compared in a previous study with three segmentation methods based on LiDAR data (Dalponte et al., 2015b) and it was shown that this method outperformed the LiDAR based methods on the delineation of broadleaf trees. This fact can also explain the very good performances of team FEM delineations in the NEON NIST data 
175 science evaluation challenge because in the study area species were mainly broadleaf or pine

176 trees. The crown shape of pine trees is quite close to the ones of many broadleaf trees.

178 Surely the choice to consider not only the position, but also the tree characteristics (i.e. height, 179 and crown radius) was the winning choice. Moreover, after the automatic matching a visual inspection of the results helped make the final improvements, as two trees were reassigned after this inspection. A visual inspection of the alignment is not doable over large datasets, even if, in our experience, it is always suggested as it helps in finding macroscopic errors. As mentioned in the introduction, the choice of alignment strategy can depend also on the type of data that can be used for this purpose. The fact that each crown delineation paper uses a different alignment method specific to the dataset is not a good approach. Indeed, there is the need to have a reference alignment method that could be used in every crown segmentation paper that allows a fair comparison among delineation results. second place. In this case the architecture that we used was effective, even if the results showed a serious problem in distinguishing minority species. This is a limitation of many other works proposed in the literature as many classifiers tend to give priority to highly represented species. A better balance in the training set could have achieved higher classification accuracies. As an example, the use of a semi-supervised classification approach (Dalponte et al., 2015a) could have improved the classification of minority species. Moreover, a feature selection specifically devoted on the identification of the best features to separate minority species cloud have helped. 
197

198

199

200

201

202

203

204

205

206

207

208

209

210

211

212

213

214

215

216

217

In this paper the results of team FEM of the NEON NIST data science evaluation challenge were

presented. The methods applied were effective as team FEM ranked first in Task 1 and 2, and second in Task 3. The delineation method proposed was based on hyperspectral images, showing that LiDAR data are not always the best data source for ITC delineation. The alignment strategy was based on both location and tree characteristics, and this combination of different information provided the added value to the perfect alignment of the crowns. The classification architecture adopted was quite standard, and it failed to classify more rare species. As a future development, it may be interesting to combine both hyperspectral and LiDAR information in the crown segmentation, and to consider classifiers, like for example semi-supervised ones, that can improve the classification of more rare species.

\section{Acknowledgements}

The National Ecological Observatory Network is a program sponsored by the National Science Foundation and operated under cooperative agreement by Battelle Memorial Institute. This material is based in part upon work supported by the National Science Foundation through the NEON Program. The ECODSE competition was supported, in part, by a research grant from NIST IAD Data Science Research Program to D.Z. Wang, E.P. White, and S. Bohlman, by the Gordon and Betty Moore Foundation's Data-Driven Discovery Initiative through grant GBMF4563 to E.P. White, and by an NSF Dimension of Biodiversity program grant (DEB1442280) to S. Bohlman. This work was partially supported by the HyperBio project (project \#244599), which was financed by the BIONÆR program of the Research Council of Norway and by TerraTec AS, Norway. 


\section{References}

219 Bruzzone L., Roli F., Serpico SB. 1995. An extension to multiclass cases of the JeffreysMatusita distance. IEEE Transactions on Geoscience and Remote Sensing 33:1318-1321.

221

222

223

224

225

226

227

Budei BC., St-Onge B., Hopkinson C., Audet F-A. 2017. Identifying the genus or species of individual trees using a three-wavelength airborne lidar system. Remote Sensing of Environment:0-1. DOI: 10.1016/j.rse.2017.09.037.

Clark ML., Roberts DA., Clark DB. 2005. Hyperspectral discrimination of tropical rain forest tree species at leaf to crown scales. Remote Sensing of Environment 96:375-398. DOI: 10.1016/j.rse.2005.03.009.

Dalponte M. 2016. Package “ itcSegment .'

Dalponte M., Bruzzone L., Gianelle D. 2008. Fusion of Hyperspectral and LIDAR Remote Sensing Data for Classification of Complex Forest Areas. IEEE Transactions on Geoscience and Remote Sensing 46:1416-1427. DOI: 10.1109/TGRS.2008.916480.

Dalponte M., Bruzzone L., Gianelle D. 2012. Tree species classification in the Southern Alps based on the fusion of very high geometrical resolution multispectral/hyperspectral images and LiDAR data. Remote Sensing of Environment 123:258-270. DOI: 10.1016/j.rse.2012.03.013.

Dalponte M., Bruzzone L., Vescovo L., Gianelle D. 2009. The role of spectral resolution and classifier complexity in the analysis of hyperspectral images of forest areas. Remote Sensing of Environment 113:2345-2355. DOI: 10.1016/j.rse.2009.06.013.

Dalponte M., Ene LT., Marconcini M., Gobakken T., Næsset E. 2015a. Semi-supervised SVM 
Dalponte M., Frizzera L., Ørka HO., Gobakken T., Næsset E., Gianelle D. 2018. Predicting stem diameters and aboveground biomass of individual trees using remote sensing data. Ecological Indicators 85:367-376. DOI: 10.1016/j.ecolind.2017.10.066.

Dalponte M., Ørka HO., Ene LT., Gobakken T., Næsset E. 2014. Tree crown delineation and tree species classification in boreal forests using hyperspectral and ALS data. Remote Sensing of Environment 140:306-317. DOI: 10.1016/j.rse.2013.09.006.

Dalponte M., Orka HO., Gobakken T., Gianelle D., Naesset E. 2013. Tree Species Classification

Dalponte M., Reyes F., Kandare K., Gianelle D. 2015b. Delineation of Individual Tree Crowns from ALS and Hyperspectral data: a comparison among four methods. European Journal of Remote Sensing 48:365-382. DOI: 10.5721/EuJRS20154821.

Duncanson LI., Dubayah RO., Cook BD., Rosette J., Parker G. 2015. The importance of spatial detail: Assessing the utility of individual crown information and scaling approaches for lidar-based biomass density estimation. Remote Sensing of Environment 168:102-112. DOI: 10.1016/j.rse.2015.06.021.

Ene L., Næsset E., Gobakken T. 2012. Single tree detection in heterogeneous boreal forests using airborne laser scanning and area-based stem number estimates. International Journal of Remote Sensing 33:5171-5193. DOI: 10.1080/01431161.2012.657363. 
Eysn L., Hollaus M., Lindberg E., Berger F., Monnet J-M., Dalponte M., Kobal M., Pellegrini M., Lingua E., Mongus D., Pfeifer N. 2015. A Benchmark of Lidar-Based Single Tree Detection Methods Using Heterogeneous Forest Data from the Alpine Space. Forests 6:1721-1747. DOI: 10.3390/f6051721.

Fassnacht FE., Latifi H., Stere??czak K., Modzelewska A., Lefsky M., Waser LT., Straub C., Ghosh A. 2016. Review of studies on tree species classification from remotely sensed data. Remote Sensing of Environment 186:64-87. DOI: 10.1016/j.rse.2016.08.013.

Ferraz A., Bretar F., Jacquemoud S., Gonçalves G., Pereira L., Tomé M., Soares P. 2012. 3-D mapping of a multi-layered Mediterranean forest using ALS data. Remote Sensing of Environment 121:210-223. DOI: 10.1016/j.rse.2012.01.020.

Hung C., Bryson M., Sukkarieh S. 2012. Multi-class predictive template for tree crown detection. ISPRS Journal of Photogrammetry and Remote Sensing 68:170-183. DOI: 10.1016/j.isprsjprs.2012.01.009.

Ke Y., Quackenbush LJ. 2011. A review of methods for automatic individual tree-crown detection and delineation from passive remote sensing. International Journal of Remote Sensing 32:4725-4747. DOI: 10.1080/01431161.2010.494184.

Lee AC., Lucas RM. 2007. A LiDAR-derived canopy density model for tree stem and crown mapping in Australian forests. Remote Sensing of Environment 111:493-518. DOI: 10.1016/j.rse.2007.04.018.

Marconi S., Graves SJ., Gong D., Nia MS., Bras M Le., Dorr J., Fontana P., Gearhart J., Greenberg C., Harris DJ., Arvind S., Nishant A., Prarabdh J., Rege S., Bohlman S., White 
EP. 2018. A data science challenge for converting airborne remote sensing data into ecological information. PeerJ Preprints.

283

284

Popescu SC., Wynne RH., Nelson RF. 2003. Measuring individual tree crown diameter with lidar and assessing its influence on estimating forest volume and biomass. In: Canadian Journal of Remote Sensing. 564-577. DOI: 10.5589/m03-027.

Pudil P., Novovičová J., Kittler J. 1994. Floating search methods in feature selection. Pattern Recognition Letters 15:1119-1125. DOI: 10.1016/0167-8655(94)90127-9.

R Development Core Team. 2008. R: A Language and Environment for Statistical Computing. $R$ Foundation for Statistical Computing 1:ISBN 3-900051-07-0.

Real R., Vargas JM. 1996. The Probabilistic Basis of Jaccard's Index of Similarity. Systematic Biology 45:380-385. DOI: 10.1093/sysbio/45.3.380.

Vauhkonen J., Ene L., Gupta S., Heinzel J., Holmgren J., Pitkänen J., Solberg S., Wang Y., Weinacker H., Hauglin KM., Lien V., Packalén P., Gobakken T., Koch B., Næsset E., Tokola T., Maltamo M. 2012. Comparative testing of single-tree detection algorithms under different types of forest. Forestry 85:27-40. DOI: 10.1093/forestry/cpr051.

Yu B., Ostland IM., Gong P., Pu R. 1999. Penalized Discriminant Analysis of In Situ Hyperspectral Data for Conifer Species Recognition. IEEE Transactions on Geoscience and Remote Sensing 37:2569-2577. 
301

302 303

304

305

306

307

308

309

310

311

312

313

314 Figure 5. Task 3: Accuracy and Specificity Scores (Per-Class).

315 Figure 6. Task 3: F1 Score (Per-Class).

316 Figure 7. Task 3: Precision (Per-Class).

317 Figure 8. Task 3: Recall (Per-Class).

\section{Tables captions}

Table 2. Task 3: overall performances.

Table 3. Task 3: confusion matrix.

\section{Figures captions}

Figure 1. Task 1: plot level confusion matrix as a bar chart.

Figure 2. Task 1: Jaccard score versus crown area. and red annotations are predicted ones. and red annotations are predicted ones.

Table 1. Task 1: overall confusion matrix. The values in the table are in square meters.

Figure 3. Task 1: the best 6 segmentations. Green annotations represent ground truth polygons,

Figure 4. Task 1: the worst 6 segmentations. Green annotations represent ground truth polygons, 


\section{Table 1 (on next page)}

Task 1: overall confusion matrix. The values in the table are in square meters. 
1

\begin{tabular}{ccc}
\hline \hline & Positive & Negative \\
\hline \hline True & 2022.8 & - \\
\hline False & 2416.6 & 1293.1 \\
\hline \hline
\end{tabular}

2

3

PeerJ Preprints | https://doi.org/10.7287/peerj.preprints.26973v1 | CC BY 4.0 Open Access | rec: 1 Jun 2018, publ: 1 Jun 2018 


\section{Table 2 (on next page)}

Task 3: overall performances. 
1

2

\begin{tabular}{ll}
\hline \hline Performance metric & Value \\
\hline \hline Cross-entropy cost & 0.8769 \\
\hline Rank-1 accuracy & 0.8800 \\
\hline Classification accuracy & 0.9809 \\
\hline Average F1 score & 0.5933 \\
\hline Average Specificity & 0.4129 \\
\hline \hline
\end{tabular}

3

4

5 


\section{Table 3 (on next page)}

Task 3: confusion matrix. 
2

\begin{tabular}{cccccccccc}
\hline \hline & ACRU & LIST & OTHER & PIEL & PIPA & PITA & QUGE & QULA & QUNI \\
\hline \hline ACRU & 0 & 0 & 0. & 0 & 1.29 & 0 & 0 & 0 & 0 \\
\hline LIST & 0 & 0.67 & 0 & 0.54 & 0 & 0 & 0 & 0 & 0 \\
\hline OTHER & 0 & 0 & 0.74 & 0 & 0 & 0 & 0 & 0 & 0 \\
\hline PIEL & 0 & 1.00 & 0.46 & 0 & 0 & 0 & 0 & 0.64 & 0 \\
\hline PIPA & 0.58 & 0 & 0 & 0 & 79.14 & 0 & 0 & 0 & 0.57 \\
\hline PITA & 0 & 0 & 0 & 0 & 2.90 & 0.85 & 0 & 0.50 & 0 \\
\hline QUGE & 0 & 0 & 0 & 0 & 0 & 0 & 0.50 & 0 & 0 \\
\hline QULA & 0 & 0 & 0 & 0 & 0 & 0 & 0 & 3.74 & 0 \\
\hline QUNI & 0 & 0 & 0 & 0 & 1.39 & 0 & 0 & 0 & 19.32 \\
\hline \hline
\end{tabular}

3

4

5 


\section{Figure 1}

Task 1: plot level confusion matrix as a bar chart.

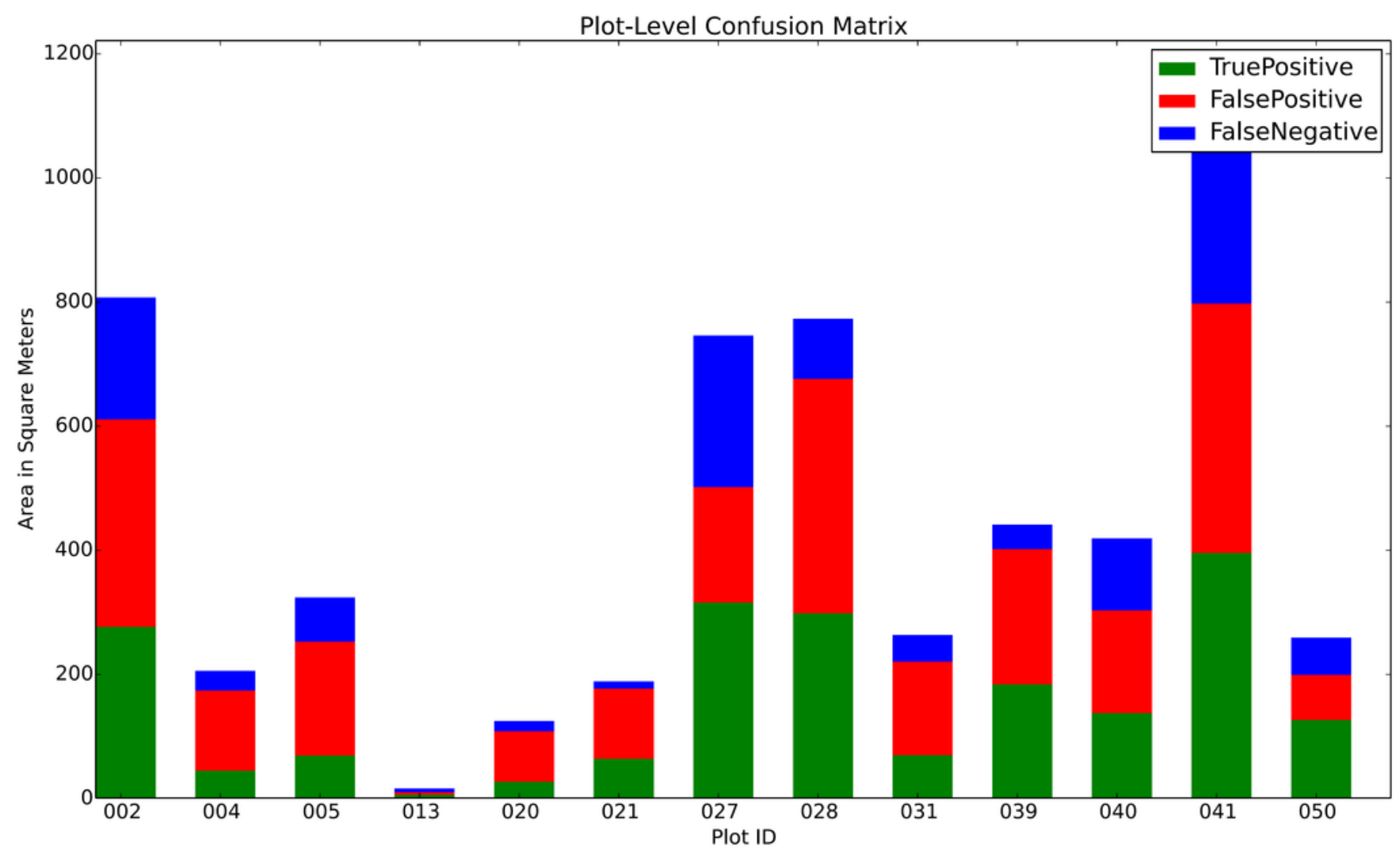


Figure 2

Task 1: Jaccard score versus crown area.

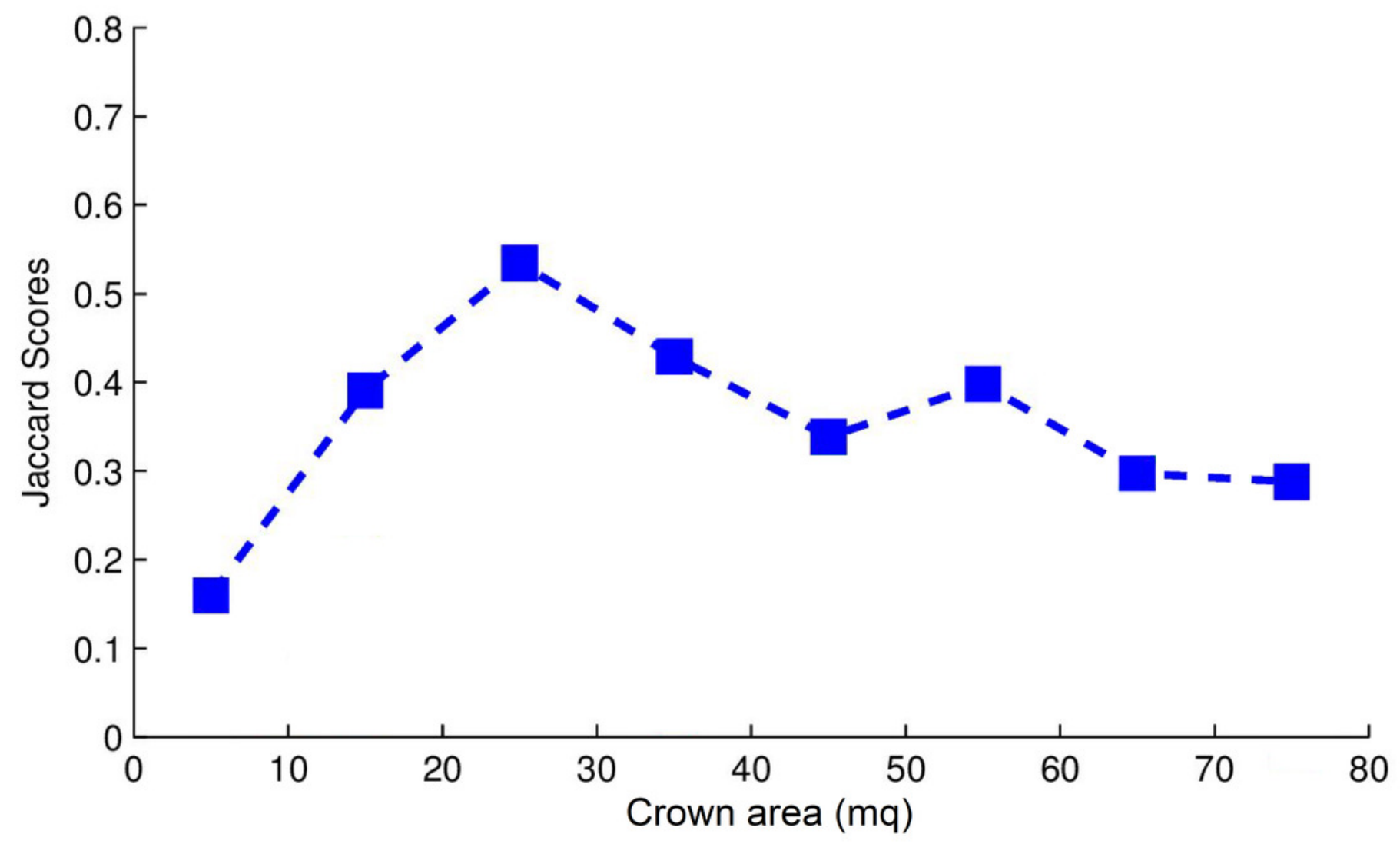


Peer Preprints $\quad$ NOT PEER-REVIEWED

Figure 3

Task 1: the best 6 segmentations. Green annotations represent ground truth polygons, and red annotations are predicted ones.
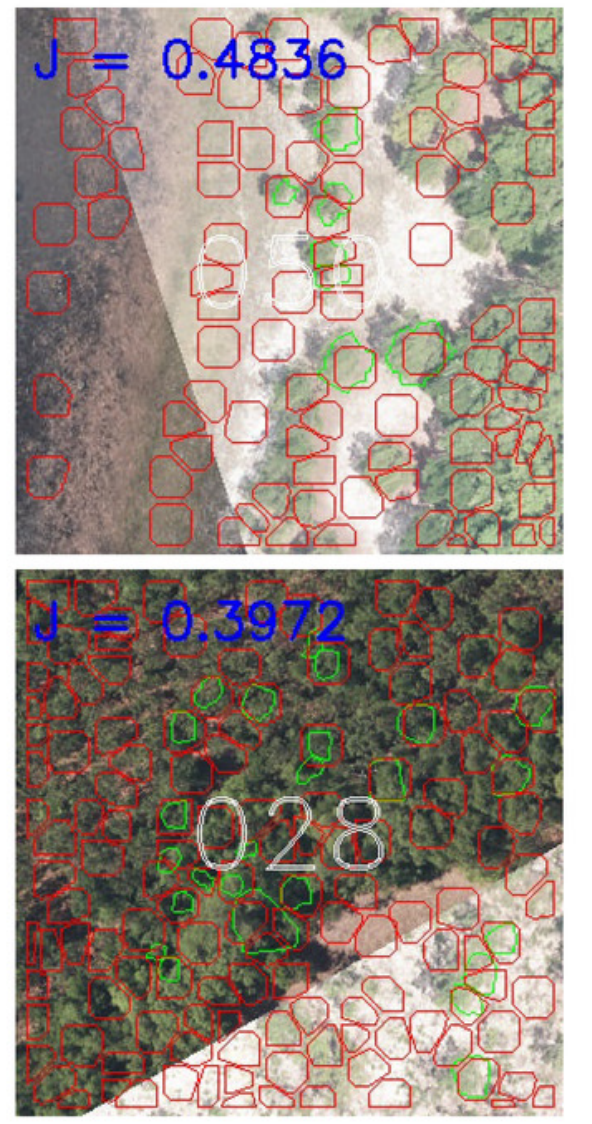
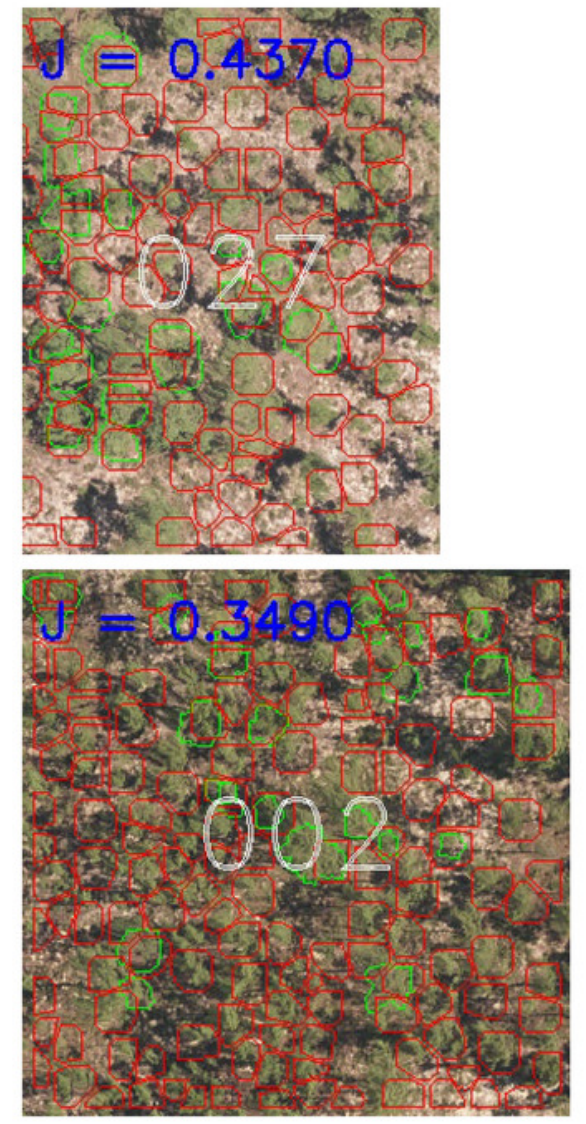
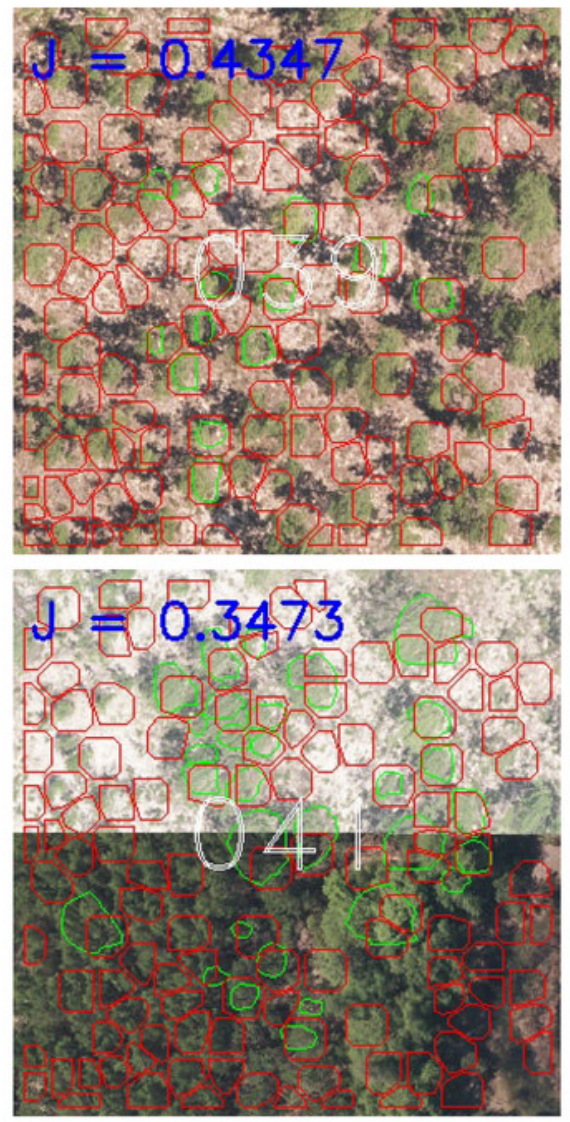


\section{Figure 4}

Task 1: the worst 6 segmentations. Green annotations represent ground truth polygons, and red annotations are predicted ones.
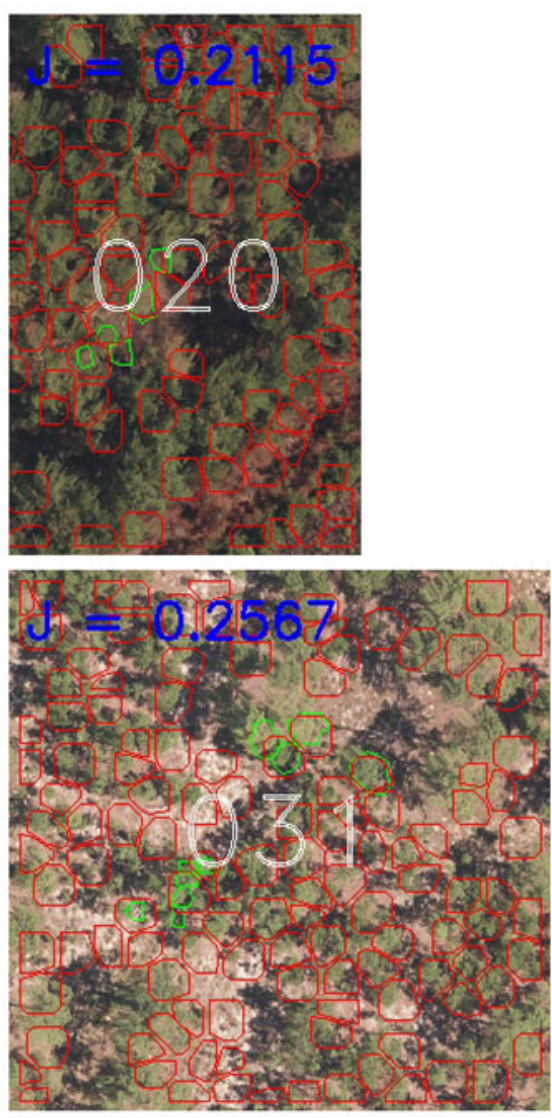
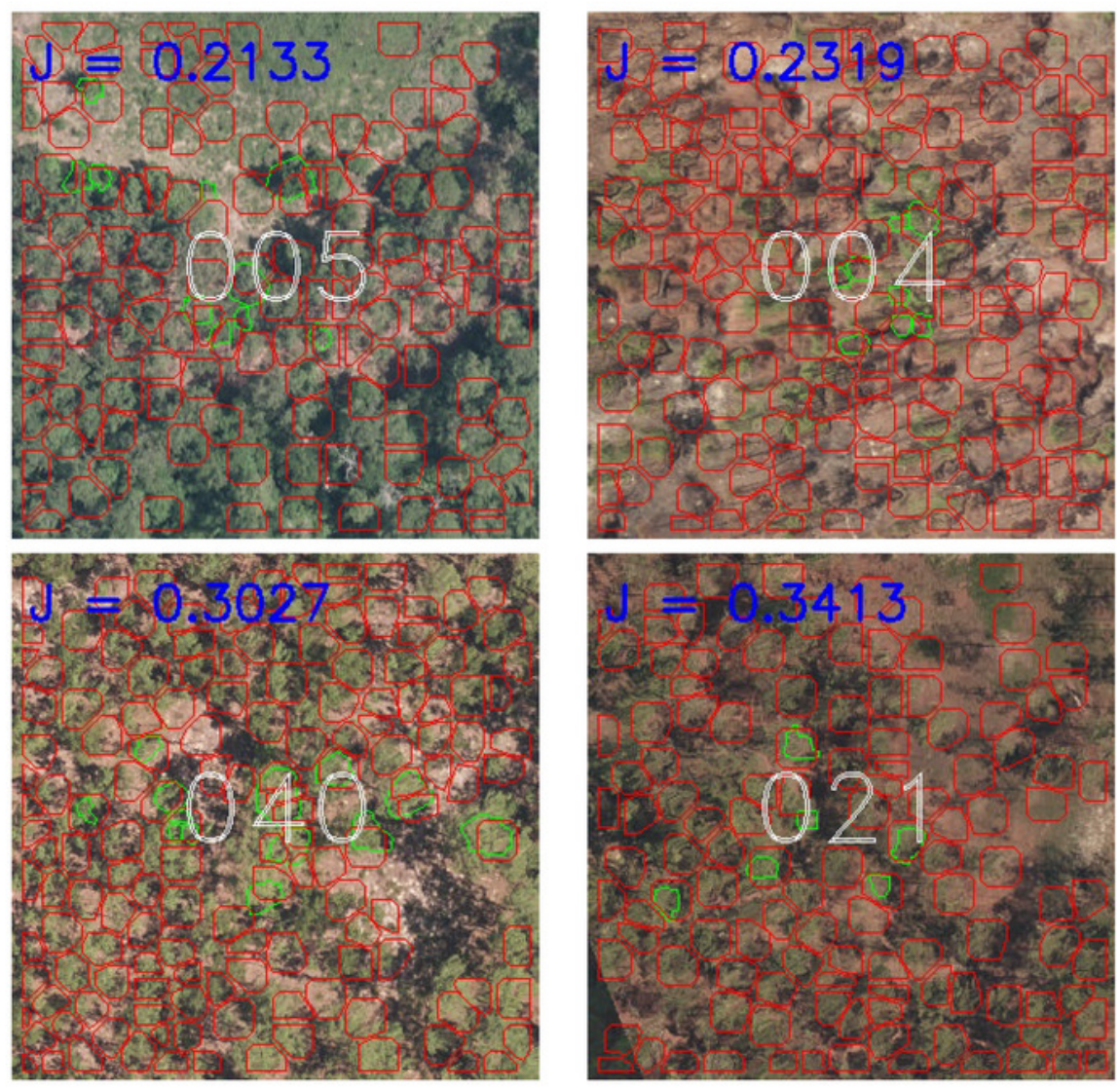
Figure 5

Task 3: Accuracy and Specificity Scores (Per-Class).

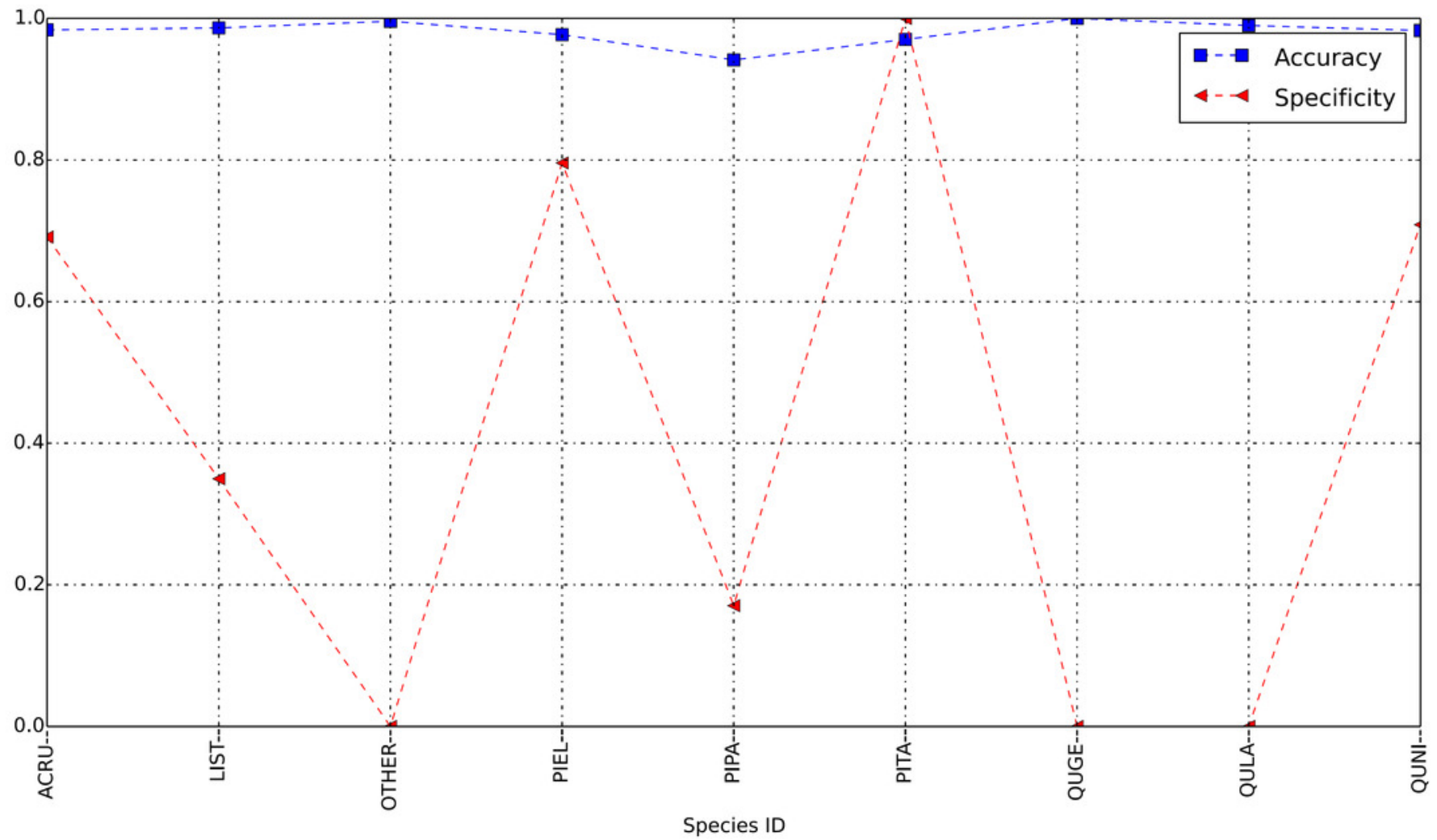




\section{Figure 6}

\section{Task 3: F1 Score (Per-Class).}

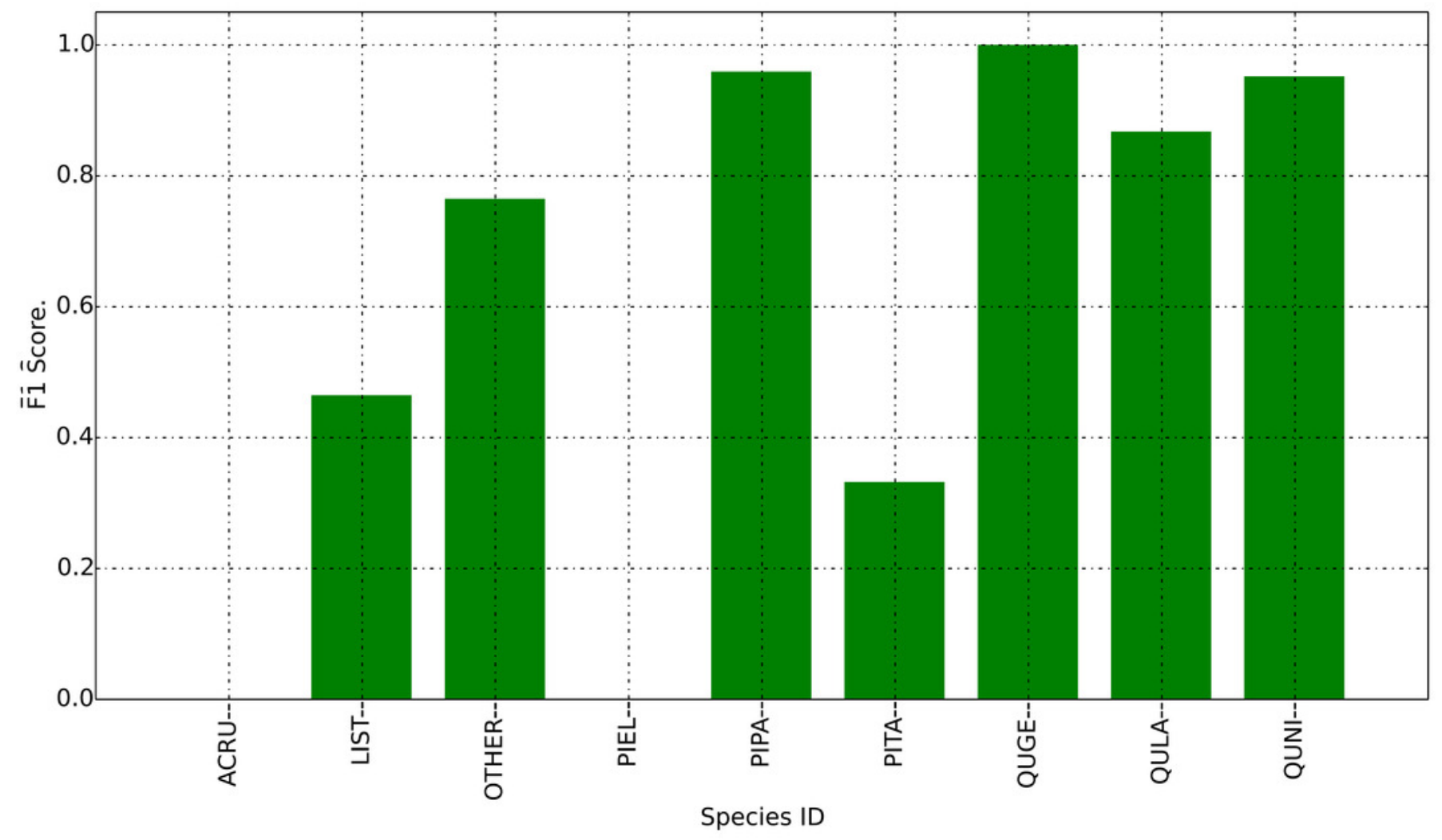




\section{Figure 7}

Task 3: Precision (Per-Class).

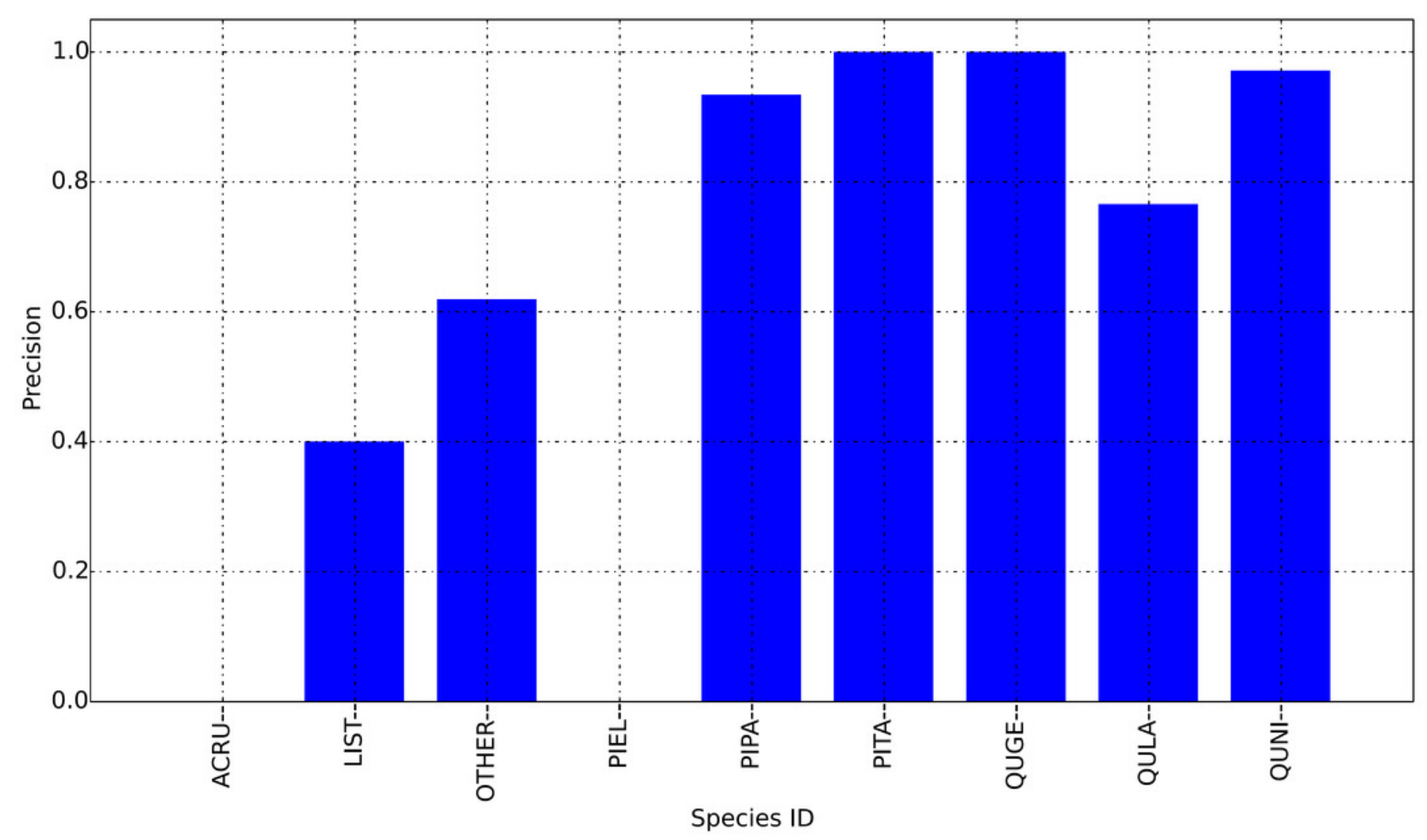


Figure 8

Task 3: Recall (Per-Class).

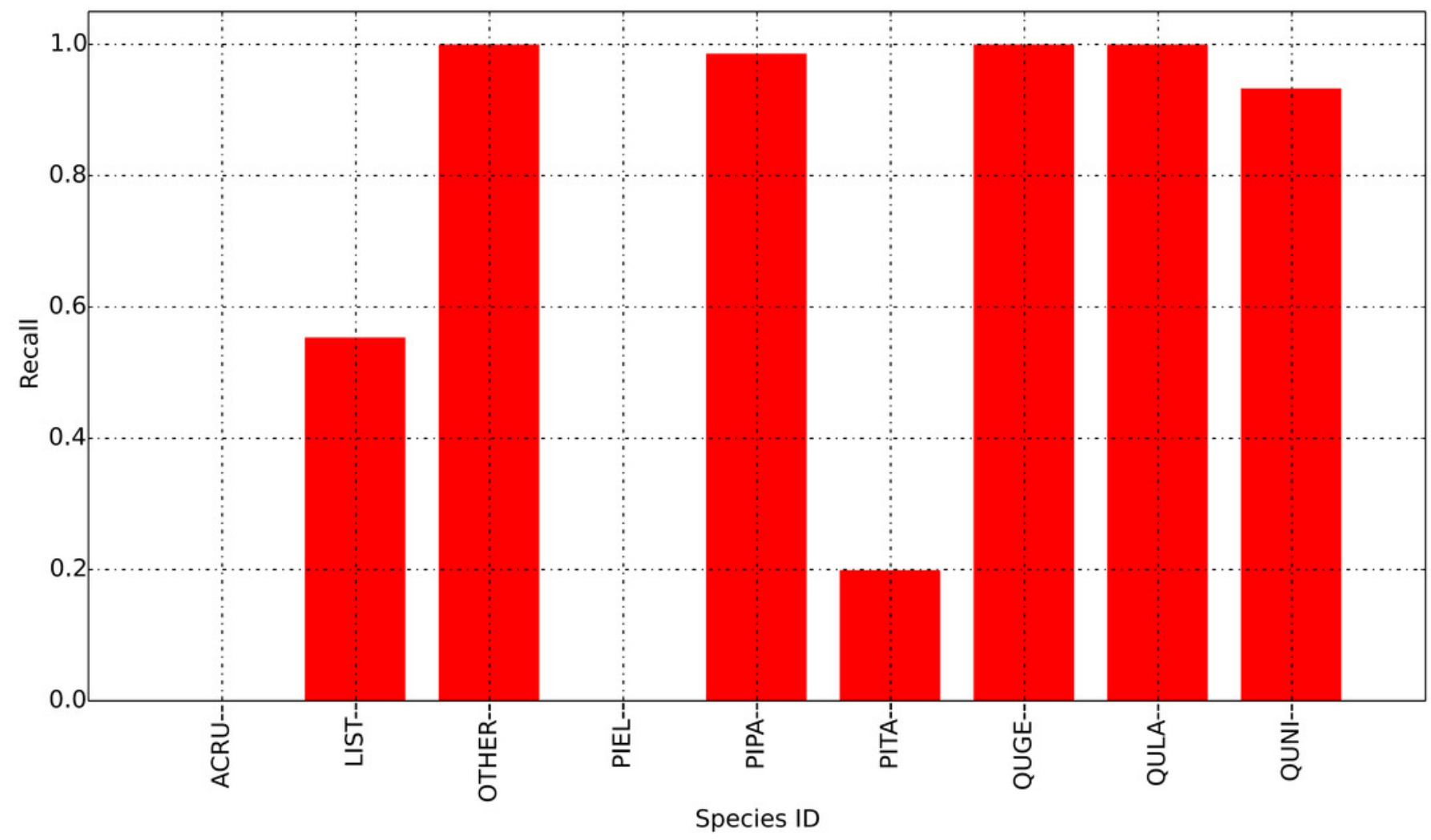

